\title{
On Consensus over Stochastically Switching Directed Topologies
}

\author{
Sundaram Vanka, Vijay Gupta and Martin Haenggi
}

\begin{abstract}
We consider average consensus algorithms executed over stochastically varying communication topologies that may be unbalanced. It is known that the state values will reach consensus, under fairly weak conditions. However, the consensus value is a random variable. We provide concentration bounds for the distance of the state vector from the consensus subspace and for the asymptotic distribution of the value to which the various nodes converge as they reach consensus. The results allow the analysis of average consensus over wireless communication networks with more realistic assumptions than before.
\end{abstract}

\section{INTRODUCTION}

Consensus algorithms are decentralized algorithms that aim at achieving agreement among all participating agents over the value of a quantity [1]. Such algorithms have seen renewed focus over the last decade [2], [3], [4], [5], [6]. Conditions for convergence of the agents to a common value are now well characterized in both deterministic [5], [6], [7] and stochastic frameworks [8], [9], [10], [11], [12], [13]. Recent work deals with characterizing the rate of convergence and imposing communication related constraints on channels between nodes. Thus, effects such as quantization [14], delays [15], additive noise [16], packet loss [17], [9] and power constraints [18] have been analyzed.

We consider the case of stochastically varying communication graphs which are of interest because they model realistic wireless communication networks. The inter-node communication in such networks is affected by probabilistic effects such as fading, interference, and channel noise. We will concentrate on the sub-class of average consensus algorithm. It is known [6] that an additional constraint for the consensus value to be the average of the values initially held by the nodes is that the communication graph be balanced at every time step. This constraint is usually imposed, e.g., by assuming a bidirectional link failure or through a perfect acknowledgement mechanism. However, this is not a realistic constraint in wireless communication.

If the graph does not remain balanced at every time step, the final value is not the average of the initial values. Instead, it is a random variable whose value depends on the set of communication graphs chosen over time. From both an analysis and a design perspective, it is important to characterize this random variable, and the rate of convergence towards the final value. In this paper, we provide

\footnotetext{
The authors are with the Department of Electrical Engineering, University of Notre Dame, Notre Dame, IN 46556 (e-mail: svanka@nd.edu, vgupta2@nd.edu, mhaenggi@nd.edu)
}

concentration bounds on the distribution of the node values at any time, and in particular, the final value.

The paper is organized as follows. We begin by formulating the problem in the next section. The node value distribution is characterized by the distribution of the values around the average and the distance from the consensus subspace. These characterizations are provided in Sections III and IV respectively. Section V presents a numerical example.

\section{PROBlem Formulation}

Consider a collection of $N$ agents (also called nodes) $\mathcal{V}=\{0,1, \ldots, N-1\}$. For each node $i$ and at any (discrete) time $t$, we specify an in-neighbor set $\mathcal{N}_{i}(t) \subseteq \mathcal{V}$ such that for any node $j \in \mathcal{N}_{i}(t)$, node $i$ has access to the value from node $j$ at time $t$. Each node $i$ has access to a number $x_{i}(0)$ at time 0 . If we denote the value held by the $i$-th node at time $t$ by $x_{i}(t)$, the (discrete-time) average consensus algorithm proceeds by each node updating its value as

$$
x_{i}(t+1)=x_{i}(t)-T_{s} \sum_{j \in \mathcal{N}_{i}(t)}\left(x_{i}(t)-x_{j}(t)\right),
$$

where $T_{s}$ is a small positive constant. If we denote this exchange of information as an edge from node $j$ to node $i$, we can define a digraph $\mathcal{G}(t)=(\mathcal{V}, \mathcal{E}(t))$ with $\mathcal{G}(t) \in \mathcal{U}$, where $\mathcal{U} \triangleq\left\{\mathcal{G}_{0}, \mathcal{G}_{1}, \ldots, \mathcal{G}_{M-1}\right\}$ is the feasible set. Graphs with self-loops are precluded. However, each node has access to its own value at every time step.

Let $\{\mathcal{G}(t)\}=\{\mathcal{G}(0), \mathcal{G}(1), \ldots\}$ be a graph-valued sequence where $\mathcal{G}(t) \in \mathcal{U}$. Defining $L_{\mathcal{G}}$ as the Laplacian of a graph $\mathcal{G}$, the consensus iteration for all nodes together is

$$
x(t+1)=W(t) x(t)
$$

where $x(t)$ is an $N \times 1$ state vector formed by stacking the values $x_{0}(t), x_{1}(t), \cdots, x_{N-1}(t)$ and $W(t) \triangleq I-$ $T_{s} L_{\mathcal{G}(t)}(I$ is the $N \times N$ identiy matrix). $W(t)$ is a stochastic matrix, irrespective of $\mathcal{G}(t)$.

We can, thus, associate with each graph-valued sequence $\{\mathcal{G}(t)\}$ a matrix-valued sequence $\{W(t)\}$ where $W(t)=$ $I-T_{s} L_{\mathcal{G}(t)}$. Therefore, starting from some known initial condition $x(0)$, one can view equation (1) as a linear iteration over $x(t)$ where the switching sequence $W(t)$ is drawn from a set $\mathcal{W}=\left\{W_{0}, W_{1}, \cdots, W_{M-1}\right\}$, with $W_{k}=I-T_{s} L_{\mathcal{G}_{k}}$. In wireless networks, whether or not a particular edge in $\mathcal{G}(t)$ exists depends on the interference and fading present in the respective wireless channel at time $t$. Since interference (at the receiver) and channel fading (between a transmitter and receiver) are stochastic 
processes, the graph $\mathcal{G}(t)$ and, in turn, the matrix $W(t)$, are random. We make the following assumptions in this paper:

- (Assumption A1) The link states, and hence the matrix sequence $W(t)$, are i.i.d across time.

- (Assumption A2) The diagonal elements $W_{i i}>0$ for all $W \in \mathcal{W}$. Defining $\bar{W} \triangleq \mathbb{E}[W]$, we also assume that the Laplacian $\bar{L}$ associated with $\bar{W}=I-T_{s} \bar{L}$ corresponds to a strongly connected weighted digraph.

- (Assumption A3) $\bar{W}$ is doubly stochastic.

Note that, contrary to [12], [13], we do not assume that the edge states (i.e., whether an edge is present or not) for different edges are independent at any given time.

Using equation (1), the state $x(t)$ can be written as

$$
x(t)=W(t-1) \cdots W(1) W(0) x(0)=\prod_{k=0}^{t-1} W(k) x(0) .
$$

Observe that $x(t)$ is a random variable whose value is determined by the stochastic process $W(t)$ and the initial state $x(0)$. Define $\bar{x}(t)$ as the expected value of $x(t)$. Using Assumption A1, we obtain for a given initial condition $x(0)$,

$$
\bar{x}(t)=\bar{W}^{t} x(0) .
$$

By Assumption A3, the average of $\bar{x}(t)$ is preserved, since

$$
\mathbf{1}^{\star} \bar{x}(t)=\mathbf{1}^{\star} \bar{W}^{t} x(0)=\mathbf{1}^{\star} x(0),
$$

where $\mathbf{1} \triangleq\left[\begin{array}{llll}1 & 1 & \ldots & 1\end{array}\right]^{\star}$ and $M^{\star}$ denotes the transpose of the matrix or the vector $M$. Moreover, $\bar{x}(t)$ converges asymptotically to the average consensus value $x_{a v}(0) 1$, where we introduce the notation $x_{a v}(t) \triangleq$ $\frac{1}{N} \sum_{n=1}^{N} x_{n}(t)$. To see this, note that

$$
\bar{x}(t)-\frac{11^{\star}}{N} x(0)=\left(\bar{W}-\frac{11^{\star}}{N}\right) \bar{x}(t-1) .
$$

Since by Assumption A2, $\bar{W}$ corresponds to a strongly connected graph, the spectral radius of $\left(\bar{W}-\frac{\mathbf{1 1}^{\star}}{N}\right)$ is strictly less than one [19], and consequently,

$$
\lim _{t \rightarrow \infty} \bar{x}(t)=\frac{\mathbf{1 1}^{\star}}{N} x(0) \triangleq x_{a v}(0) 1 .
$$

It was also shown recently [11] that that $x(t)$ converges almost surely to some consensus point if $\mathbf{A} \mathbf{2}$ holds, i.e.,

$$
\lim _{t \rightarrow \infty} x(t)=\alpha \mathbf{1}
$$

almost surely. We now build on a geometric interpretation from [11], and introduce notation that will be used later. Let $\|v\|$ denote the Euclidean norm of vector $v$. We can write

$\left\|x(t)-\mathbf{1} x_{a v}(0)\right\|^{2}=\left\|x(t)-\mathbf{1} x_{a v}(t)+\mathbf{1} x_{a v}(t)-\mathbf{1} x_{a v}(0)\right\|^{2}$.

Expanding the right hand side yields

$$
\begin{aligned}
\left\|x(t)-\mathbf{1} x_{a v}(0)\right\|^{2}=\left\|x(k)-\mathbf{1} x_{a v}(t)\right\|^{2} \\
+\left\|\mathbf{1} x_{a v}(t)-\mathbf{1} x_{a v}(0)\right\|^{2} \\
+2\left\langle x(t)-\mathbf{1} x_{a v}(t), \mathbf{1} x_{a v}(t)-\mathbf{1} x_{a v}(0)\right\rangle,
\end{aligned}
$$

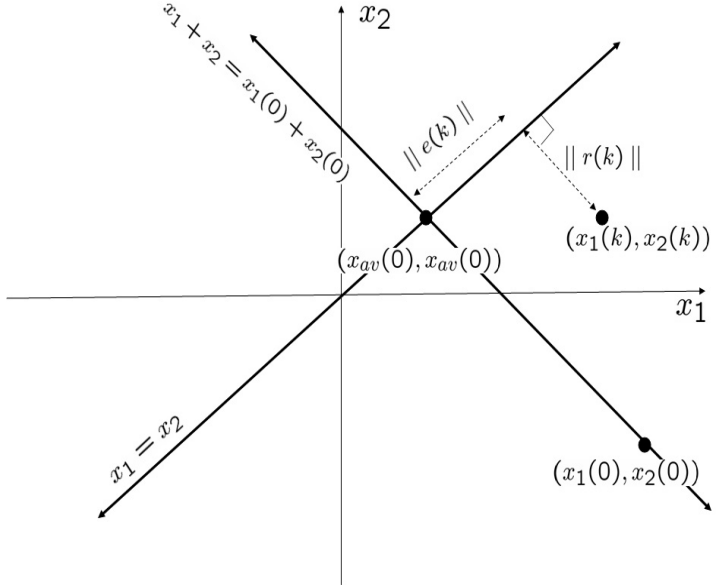

Fig. 1. Geometric interpretation of equation (4) for $N=2$. The average consensus point is the intersection of the straight lines $x_{1}+x_{2}=$ $x_{1}(0)+x_{2}(0)$ with the straight line $x_{1}=x_{2}$, shown by the point $\left(x_{a v}(0), x_{a v}(0)\right)$. We find concentration bounds on the distributions of $r$ and $e$ for all $t \geq 0$.

where $\langle.,$.$\rangle denotes the standard inner product in \mathbb{R}^{N}$. This inner product, in turn, simplifies as

$$
\begin{aligned}
& \left\langle x(t)-\mathbf{1} x_{a v}(t), \mathbf{1} x_{a v}(t)-\mathbf{1} x_{a v}(0)\right\rangle \\
& =\left\langle x(t)-\mathbf{1} x_{a v}(t), \mathbf{1}\right\rangle x_{a v}(t)-\left\langle x(t)-\mathbf{1} x_{a v}(t), \mathbf{1}\right\rangle x_{a v}(0) \\
& =N x_{a v}^{2}(t)-N x_{a v}^{2}(t)-N x_{a v}(t) x_{a v}(0)+N x_{a v}(t) x_{a v}(0) \\
& =0 .
\end{aligned}
$$

Thus, we obtain

$$
\begin{aligned}
& \left\|x(t)-\mathbf{1} x_{a v}(0)\right\|^{2} \\
& =\left\|x(t)-\mathbf{1} x_{a v}(t)\right\|^{2}+\left\|\mathbf{1} x_{a v}(t)-\mathbf{1} x_{a v}(0)\right\|^{2} \\
& =\|r(t)\|^{2}+\|e(t)\|^{2},
\end{aligned}
$$

where $r(t) \triangleq x(t)-\mathbf{1} x_{a v}(t)$ and $e(t) \triangleq \mathbf{1} x_{a v}(t)-\mathbf{1} x_{a v}(0)$.

A geometric interpretation of this result is shown in Figure 1 for the case of two agents. In the state space of the agent values, the consensus subspace is the straight line $x_{1}=x_{2}=\cdots=x_{N}$. The term $\|r(t)\|$ represents the (residual) distance of $x(t)$ from its closest point in the consensus subspace, while $\|e(t)\|$ represents the distance from this closest point and the average consensus point.

\section{Specific Problems Considered in this Work}

Equating the infinity-norms on both sides in (1) yields

$$
\begin{aligned}
\|x(t)\|_{\infty} & =\|W(t-1) x(t-1)\|_{\infty} \\
& \leq\|W(t-1)\|_{\infty}\|x(t-1)\|_{\infty}=\|x(t-1)\|_{\infty},
\end{aligned}
$$

where we exploit the sub-multiplicativity of the infinitynorm, and the fact that $W(t)$ is stochastic. Through a recursive application of this result, we obtain $\|x(t)\|_{\infty} \leq$ $\|x(0)\|_{\infty}$. Thus, a straightforward application of the Dominated Convergence Theorem [20] allows us to interchange the integral and the limit and write $\mathbb{E}\left[\lim _{t \rightarrow \infty} x(t)\right]=$ $\lim _{t \rightarrow \infty} \mathbb{E}[x(t)]$. In light of (3) and (2), this simplifies to 
$\mathbb{E}[\alpha]=x_{a v}(0)$. Thus, the expectation of the final state remains the average consensus point. In this paper, we extend this result by answering the following questions:

1) What is the distribution of $\alpha$ ?

2) Can we characterize the distance $r(t)$ of the state $x(t)$ from the consensus subspace at any time $t$ given an initial state $x(0)$ and a random switching sequence $\{W(t)\}$ ?

\section{ASYMPTOTIC DisTRIBUTION}

As shown above, as $t \rightarrow \infty, x(t)$ converges to a point $\alpha \mathbf{1}$ on the convergence subspace, where $\alpha$ is a scalar random variable. To find the entire distribution of $\alpha$, we need to characterize $e(t)=\mathbf{1}\left(x_{a v}(t)-x_{a v}(0)\right)$. We can obtain a quantity that is slightly easier to characterize by rewriting

$$
\begin{aligned}
e(t) & =\frac{\mathbf{1}}{N}\left(\mathbf{1}^{\star} x(t)-\mathbf{1}^{\star} x(0)\right) \\
& =\left(\frac{\mathbf{1}^{\star} \prod_{l=0}^{t-1} W(l) x(0)-\mathbf{1}^{\star} x(0)}{N}\right) \mathbf{1} \triangleq \delta(t) \mathbf{1} .
\end{aligned}
$$

Since the sequence $\{W(t)\}$ is uncorrelated and $\bar{W}$ is doubly stochastic,

$$
\mathbb{E} \delta(t)=\frac{\mathbf{1}^{\star}\left(\bar{W}^{t}-I\right) x(0)}{N}=0 .
$$

Before proving the concentration result, we prove two preliminary results and introduce some notation. The first result provides a partial characterization of the distribution of the weight matrix $W(t)$ at any time $t$.

Lemma 3.1: Denote the $(i, j)$-th element of $\bar{W}$ by $\eta_{i j}$. For any $t \geq 0$, if $\mathcal{G}_{m} \in \mathcal{U}$ is chosen with probability $p_{m}(t)$

$$
\|W(t)-\bar{W}\|_{\infty} \leq 2 T_{s} \max _{i}\left(\sum_{j \neq i}\left(1-\eta_{i j}\right), \sum_{j \neq i} \eta_{i j}\right) .
$$

Proof: Let $X_{i j}(t)(i \neq j)$ be a random variable which equals one when there is a directed link from $j$ to $i$ at time $t$, and zero otherwise. Thus, $X_{i j}(t)$ is Bernoulli with parameter $\eta_{i j}(t)$ where

$$
\eta_{i j}(t)=\sum_{m=0}^{M-1} 1_{\mathcal{E}_{i j} \in \mathcal{G}_{m}} p_{m}(t)
$$

Define

$$
\begin{aligned}
X_{i i}(t) & \triangleq \sum_{j \neq i} X_{i j}(t) \\
\eta_{i i}(t) & \triangleq \mathbb{E} X_{i i}(t)=\sum_{j \neq i} \eta_{i j}(t) .
\end{aligned}
$$

Therefore $W(t)$ is a random matrix whose entries are $W_{i j}(t)=\Delta_{i j}-T_{s} X_{i j}(t)$, where $\Delta_{i j}$ is a Kronecker delta.
We thus obtain

$$
\begin{aligned}
\|W(t)-\bar{W}\|_{\infty} & =T_{s} \max _{i}\left(\sum_{j}\left|W_{i j}-\bar{W}_{i j}\right|\right) \\
& =T_{s} \max _{i}\left(\left|X_{i i}-\eta_{i i}\right|+\sum_{j \neq i}\left|X_{i j}-\eta_{i j}\right|\right) \\
& =T_{s} \max _{i} f_{i}\left(\left\{X_{i j}\right\}\right),
\end{aligned}
$$

where

$$
f_{i} \triangleq\left|\sum_{j \neq i}\left(X_{i j}-\eta_{i j}\right)\right|+\sum_{j \neq i}\left|X_{i j}-\eta_{i j}\right|
$$

is a function on $\{0,1\}^{N}$. For a fixed value of $i$, suppose that $X_{i j}$ 's in some index set $j \in \mathcal{I} \subseteq \mathcal{N} \backslash\{i\}$ assume the value one and for values of $j$ outside this index set assume the value zero. Then, we can write

$$
f_{i}\left(\left\{X_{i j}\right\}\right)=|| \mathcal{I}\left|-\sum_{j \neq i} \eta_{i j}\right|+|\mathcal{I}|-\sum_{j \in \mathcal{I}} \eta_{i j}+\sum_{j \in \mathcal{N} \backslash \mathcal{I}} \eta_{i j} .
$$

Define $Y=|\mathcal{I}|-\sum_{j \neq i} \eta_{i j}$. If $Y \geq 0$ then we obtain

$$
f_{i}\left(\left\{X_{i j}\right\}\right)=2 \sum_{j \in \mathcal{I}}\left(1-\eta_{i j}\right) \leq 2 \sum_{j \neq i}\left(1-\eta_{i j}\right) .
$$

Similarly, if $Y<0$, then we obtain

$$
f_{i}\left(\left\{X_{i j}\right\}\right)=2 \sum_{j \in \mathcal{N} \backslash\{\mathcal{I}\}} \eta_{i j} \leq 2 \sum_{j \neq i} \eta_{i j} .
$$

The result follows by substituting (6) and (7) in (5). For future reference, define

$$
C_{1}=2 T_{s} \max _{i}\left(\sum_{j \neq i}\left(1-\eta_{i j}\right), \sum_{j \neq i} \eta_{i j}\right) .
$$

Lemma 3.2: Denote the eigenvalues of $\bar{W}$ by $1=\lambda_{0}>$ $\lambda_{1} \geq \cdots \geq \lambda_{N-1}>-1$ and let $\bar{W}=S^{-1} \Lambda S$ be its spectral decomposition. Let $s_{i}$ denote the $i^{\text {th }}$ (normalized) eigenvector, and $v_{i}=\left[\begin{array}{llll}v_{i}^{(0)} & v_{i}^{(1)} & \ldots & v_{i}^{(N-1)}\end{array}\right]$ the $i^{\text {th }}$ row of $S^{-1}$. If $\bar{W}$ is normal or has no repeated eigenvalues, then for any $t \geq 0$, we have

$$
\left\|\bar{W}^{t}-\frac{\mathbf{1 1}^{\star}}{N}\right\|_{\infty} \leq C_{2} \mu^{t}
$$

where

$$
\begin{gathered}
\mu \triangleq \max _{i=1,2, \cdots N-1}\left|\lambda_{i}\right| \\
C_{2} \triangleq \sum_{i=1}^{N-1} \sum_{j=0}^{N-1}\left|v_{i}^{(j)}\right| .
\end{gathered}
$$

Proof: From the spectral decomposition we see that for any positive integer $t, \bar{W}=S^{-1} \Lambda S$ implies $\bar{W}^{t}=$ $S^{-1} \Lambda^{t} S$. We now expand the latter expression and use the fact that $\bar{W}$ is doubly stochastic, and will therefore have $1^{\star}$ as a left eigenvector with eigenvalue 1 , to obtain

$$
\bar{W}^{t}=N^{-1} \mathbf{1 1}^{\star}+\sum_{i=1}^{N-1} \lambda_{i}^{t} s_{i} v_{i}^{\star} .
$$


Define $A=\bar{W}^{t}-N^{-1} \mathbf{1} 1^{\star}$, and denote the $(m, n)$-th element of $A$ by $a_{m n}$. Therefore,

$$
\begin{aligned}
\|A\|_{\infty} & =\max _{m} \sum_{n=0}^{N-1}\left|a_{m, n}\right| \\
& =\max _{m} \sum_{n=0}^{N-1}\left|\sum_{i=1}^{N-1} \lambda_{i}^{t} s_{i}^{(m)} v_{i}^{(n)}\right| \\
& \leq \sum_{n=1}^{N} \max _{\lambda_{i} \neq 1}\left|\lambda_{i}^{t}\right| \sum_{i=1}^{N-1}\left|v_{i}^{(n)}\right| \\
& \leq \underbrace{\max _{\lambda_{i} \neq 1}\left|\lambda_{i}\right|^{t}}_{\mu^{t}} \underbrace{\sum_{i=1}^{N-1} \sum_{n=0}^{N-1}\left|v_{i}^{(n)}\right|}_{C_{2}}
\end{aligned}
$$

This result can be extended to $\bar{W}$ that is not normal and has repeated eigenvalues. Assume that $\bar{W}$ has $p$ (possibly repeated) eigenvalues $\lambda_{0}=1, \lambda_{1}, \lambda_{2}, \ldots, \lambda_{p}$ with multiplicity $1, r_{1}, \ldots, r_{p}$ respectively. Denote $r^{\prime}=\max \left(\left\{r_{i}\right\}\right)-1$. We can use a Jordan decomposition to obtain

$$
\bar{W}^{t}=\frac{\mathbf{1 1}^{\star}}{N}+\sum_{i=1}^{N-1} \sum_{l=1}^{r_{i}} \sum_{m=1}^{l}\left(\begin{array}{c}
t \\
l-m
\end{array}\right) \lambda_{i}^{t+m} s_{i, m} v_{i, l}^{\star},
$$

where $s_{i, j}$ denotes the $j^{\text {th }}$ (generalized) eigenvector of the $i^{\text {th }}$ eigenvalue and $v_{i, j}$ denotes the corresponding row in $S^{-1}$. Using similar arguments as above, we can prove that

$$
\left\|\bar{W}^{t}-N^{-1} \mathbf{1} \mathbf{1}^{\star}\right\|_{\infty} \leq C_{2}\left(\begin{array}{c}
t \\
r^{\prime}
\end{array}\right) \mu^{\left(t-r^{\prime}\right)} .
$$

For sake of notational simplicity, unless otherwise stated, we will assume that $\bar{W}$ is normal or has distinct eigenvalues. The results presented below can be extended to more general cases, and we will mention briefly the general statements towards the end.

The main result in this section characterizes the distribution of the projection of the state $x(t)$ on to the consensus space from the average consensus point as follows.

Proposition 3.3: Consider the problem formulation as posed in Section II with an initial condition $x(0)$. Then the distribution of $\delta(t)$ at any time $t \geq 0$ satisfies

$$
\mathbb{P}(|\delta(t)| \geq \epsilon) \leq \min \left\{1,2 \exp \left(-\epsilon^{2} \beta(t)\right)\right\}
$$

where

$$
\beta(t) \triangleq \frac{\epsilon^{2}\left(1-\mu^{2}\right)}{2 C^{2}\|x(0)\|_{\infty}^{2}\left(1-\mu^{2 t}\right)}
$$

and $C \triangleq C_{1} C_{2}$ with $C_{1}$ as defined in Lemma 3.1, and $C_{2}$ and $\mu$ as defined in Lemma 3.2.

Proof: The value of $\delta(t)$ depends on the choices $W(0)$, $\cdots, W(t-1)$. Denote the conditional expectation of $\delta(t)$ given the past $k$ values $W(t-k), \cdots, W(t-1)$ as

$$
Z_{k}(t) \triangleq \mathbb{E}[\delta(t) \mid\{W(t-1) \ldots, W(t-k)\}] .
$$

From the definition,

$$
Z_{k}(t)=\frac{\mathbf{1}^{\star}\left(W(t-1) \cdots W(t-k) \bar{W}^{t-k} x(0)-x(0)\right)}{N} .
$$

In particular, $Z_{0}(t)=0$ and $Z_{t}(t)=\delta(t)$. By construction, the sequence $\left\{Z_{k}(t)\right\}$ is a martingale in $k$ for a given $t$ [21]. We aim to bound the increase between successive steps of the martingale $\left\{Z_{k}(t)\right\}$. From the definition,

$$
\begin{aligned}
\left|Z_{k}(t)-Z_{k-1}(t)\right|= & \mid N^{-1} \mathbf{1}^{\star} W(t-1) \cdots W(t-k+1) \\
& (W(t-k)-\bar{W}) \bar{W}^{t-k} x(0) \mid .
\end{aligned}
$$

Using the spectral expansion

$$
\bar{W}=\frac{11^{\star}}{N}+\sum_{i=2}^{N} \lambda_{i} s_{i} v_{i}^{\star}
$$

and the fact that $W(t)$ is always stochastic, we obtain

$$
(W(t-k)-\bar{W}) \bar{W}^{t-k}=(W(t-k)-\bar{W}) \sum_{i=2}^{N} \lambda_{i}^{t-k} s_{i} v_{i}^{\star} .
$$

Using this result in (8), we obtain

$$
\begin{aligned}
& \left|Z_{k}(t)-Z_{k-1}(t)\right| \\
& \left.=\mid N^{-1} \mathbf{1}^{\star} W(t-1) \cdots W(t-k+1)\right)(W(t-k)-\bar{W}) \\
& \qquad \sum_{i=2}^{N}\left(\lambda_{i}^{t-k} s_{i} v_{i}^{\star}\right) x(0) \mid \\
& \leq\left\|N^{-1} \mathbf{1}^{\star}\right\|_{\infty}\|W(t-1) \cdots W(t-k+1)\|_{\infty} \\
& \|W(t-k)-\bar{W}\|_{\infty}\left\|\sum_{i=2}^{N} \lambda_{i}^{t-k} s_{i} v_{i}^{\star}\right\|_{\infty}\|x(0)\|_{\infty},
\end{aligned}
$$

where $\|.\|_{\infty}$ denotes the $\infty$-norm. We now upper bound the right hand side. It is easy to verify that $\left\|N^{-1} \mathbf{1}\right\|_{\infty}=1$. Moreover, since each $W(k)$ is stochastic, and the $\infty$-norm is sub-multiplicative, we have

$$
\|W(t-1) \cdots W(t-k+1)\|_{\infty} \leq \prod_{l=1}^{k-1}\|W(t-l)\|_{\infty} \leq 1 .
$$

Upper bounds for the third and the fourth terms have already been obtained in Lemma 3.1 and Lemma 3.2 respectively. For a given $x(0)$, we thus obtain

$$
\left|Z_{k}(t)-Z_{k-1}(t)\right| \leq C_{1} C_{2} \mu^{t-k}\|x(0)\|_{\infty} .
$$

This allows us to use Azuma's inequality [21] to obtain

$$
\begin{aligned}
\mathbb{P}\left(\left|Z_{t}(t)-Z_{0}(t)\right| \geq \epsilon\right) & \leq 2 \exp \left(\frac{-\epsilon^{2}}{2 C^{2}\|x(0)\|_{\infty}^{2} \gamma(t)}\right) \\
& =2 \exp \left(-\beta(t) \epsilon^{2}\right),
\end{aligned}
$$

where

$$
\begin{aligned}
\gamma(t) & =\sum_{k=0}^{t} \mu^{2(t-k)}=\frac{1-\mu^{2 t}}{1-\mu^{2}} \\
C & =C_{1} C_{2} \\
\beta(t) & =\frac{1}{2 C^{2}\|x(0)\|_{\infty}^{2} \gamma(t)} .
\end{aligned}
$$


Since the measure under consideration is probability (thus always bounded by unity), the result follows.

Since $Z_{t}(t)-Z_{0}(t)=\delta(t)$, the result can be interpreted as a concentration result (about the mean) for the probability distribution function of $e(t)$ for all $t$. As a special case, we obtain the asymptotic distribution as $t \rightarrow \infty$.

Corollary 3.4: The asymptotic distribution of $\alpha$ obeys the inequality

$$
\begin{aligned}
\mathbb{P}(|\alpha| \geq \epsilon) \leq 2 \exp \left(-\beta(\infty) \epsilon^{2}\right) \\
=2 \exp \left(-\frac{\epsilon^{2}\left(1-\mu^{2}\right)}{2 C^{2}\|x(0)\|_{\infty}^{2}}\right) .
\end{aligned}
$$

Proof: Since $x(t)$ converges to consensus almost surely, the asymptotic distribution is obtained by taking the limit $t \rightarrow \infty$ in the result obtained in Proposition 3.3.

We conclude this section with two remarks. To begin with, note that we can utilize (10) to bound any moment of $\alpha$ by using the following identity [20, p. 198] for a random variable $X$ defined over a probability space $(\Omega, \mathcal{F}, \mu)$ :

$$
\mathbb{E}|X|^{r}=\int|X|^{r} d \mu \equiv r \int_{0}^{\infty} u^{r-1} \lambda(u) d u .
$$

where $r>0$ and $\lambda(u) \triangleq \mathbb{P}(|X| \geq u)$. In our case, Proposition 3.3 provides a function $\eta(u)$ that upper bounds $\lambda(u)$ at every point. Thus, we can calculate

$$
\mathbb{E}|X|^{r} \leq r \int_{0}^{\infty} u^{r-1} \eta(u) d u .
$$

As an example, we can bound the asymptotic variance as

$$
\begin{aligned}
\lim _{t \rightarrow \infty} \mathbb{E}|\delta(t)|^{2} & \leq 2 \int_{0}^{\infty} u \exp \left(-u^{2} \beta(\infty)\right) d u \\
& =\beta^{-1}(\infty)=\frac{2 C^{2}\|x(0)\|_{\infty}^{2}}{1-\mu^{2}} .
\end{aligned}
$$

Our second remark concerns the case where $\bar{W}$ may have repeated eigenvalues and is not normal. Using similar arguments as for the case of non-repeated eigenvalues, we can obtain a similar bound as in (10) but with

$$
\gamma(t)=\mu^{-2 r^{\prime}} \sum_{k=0}^{t}\left(\begin{array}{l}
k \\
r^{\prime}
\end{array}\right)^{2} \mu^{2 k}
$$

As $t \rightarrow \infty$, this converges to a hypergeometric series [22]

$$
\lim _{t \rightarrow \infty} \sum_{k=0}^{t}\left(\begin{array}{l}
k \\
r^{\prime}
\end{array}\right)^{2} \mu^{2 k}={ }_{3} F_{2}\left(1,1,1 ;-r^{\prime}+1,-r^{\prime}+1 ; \mu^{2}\right) \text {, }
$$

where

$$
{ }_{m} F_{n}\left(a_{1}, a_{2} \ldots, a_{m} ; b_{1}, b_{2} \ldots, b_{n} ; z\right) \triangleq \sum_{l=0}^{\infty} \frac{\alpha_{l} z^{l}}{l !}
$$

with $\alpha_{0}=1$ and

$$
\frac{\alpha_{l+1}}{\alpha_{l}}=\frac{\prod_{i=1}^{m}\left(l+a_{i}\right)}{\prod_{j=1}^{n}\left(l+b_{j}\right)} z
$$

\section{Convergence to Consensus Subspace}

We now use similar techniques as above to provide concentration bounds on the distance $r(t)$ of the vector $x(t)$ from the consensus subspace at any time $t$. We can write

$$
\begin{aligned}
r(t) & =x(t)-\mathbf{1} x_{a v}(t) \\
& =\left(I-N^{-1} \mathbf{1 1}\right) x(t) \\
& =P W(t-1) W(t-2) \cdots W(0) x(0),
\end{aligned}
$$

where $P=I-N^{-1} \mathbf{1} \mathbf{1}^{\star}$ is the projection operator. Once again, since $\{W(t)\}$ is uncorrelated, $\mathbb{E} r(t)=P \bar{W}^{t} x(0)$. The martingale $\left\{Y_{k}(t)\right\}$ in this case is given by

$$
\begin{aligned}
Y_{k}(t) & =\mathbb{E}[r(t) \mid\{W(t-1), W(t-2), \ldots W(t-k)\}] \\
& =P W(t-1) W(t-2) \cdots W(t-k) \bar{W}^{t-k} x(0) .
\end{aligned}
$$

In particular, $Y_{0}(t)=P \bar{W}^{t} x(0)$ and $Y_{t}(t)=r(t)$. To bound the increase in successive steps of the martingale, consider

$$
\begin{aligned}
& \left\|Y_{k}(t)-Y_{k-1}(t)\right\| \\
& =\| P W(t-1) \cdots W(t-k+1) \\
& \quad(W(t-k)-\bar{W}) \bar{W}^{t-k} x(0) \|_{\infty} \\
& \leq\|P\|_{\infty}\|W(t-1) \cdots W(t-k+1)\|_{\infty} \\
& \quad\left\|(W(t-k)-\bar{W}) \bar{W}^{t-k} x(0)\right\|_{\infty},
\end{aligned}
$$

by using the sub-multiplicative property of the $\infty$-norm. Since $P$ is the projection operator onto the one-dimensional consensus subspace, $\|P\|_{\infty}=1$. For the rest of the terms, a similar approach as in equation (8) in Section III yields

$$
\left\|Y_{k}(t)-Y_{k-1}(t)\right\|_{\infty} \leq C \mu^{t-k}\|x(0)\|_{\infty} .
$$

where $C$ and $\mu$ are as defined earlier. Applying a vector version of Azuma's inequality we obtain

$$
\mathbb{P}\left(\left\|r(t)-P \bar{W}^{t} x(0)\right\|_{\infty} \geq \epsilon\right) \leq 2 \exp \left(-\epsilon^{2} \alpha(t)\right) .
$$

This result bounds the distribution of $r(t)$ about a deterministic evolution $P \bar{W}^{t} x(0)$. We summarize this result below.

Proposition 4.1: Consider the average consensus problem with the assumptions as stated in Section II. The state vector $x(t)$ converges almost surely to a point $\alpha \mathbf{1}$ on the consensus subspace. Then the distance $r(t)$ of the state from the consensus subspace has a mean $P \bar{W}^{t} x(0)$ and satisfies

$$
\mathbb{P}\left(\left\|r(t)-P \bar{W}^{t} x(0)\right\|_{\infty} \geq \epsilon\right) \leq 2 \exp \left(-\epsilon^{2} \beta(t)\right) .
$$

where $\beta(t)$ is as defined in Section III.

The concentration inequalities in Propositions 3.3 and 4.1 can be used to probabilistically bound $x(t)$ in the state space using equation (4) for any given $x(0)$.

\section{Numerical Results}

We now present some simulation results for the distribution of $\alpha$ in a simple network. To illustrate our results, we consider the simplest network possible with $N=2$ nodes that run the average consensus algorithm in the presence of packet losses. In simulations, these presence of drops are modeled as link states being Bernoulli random variables 


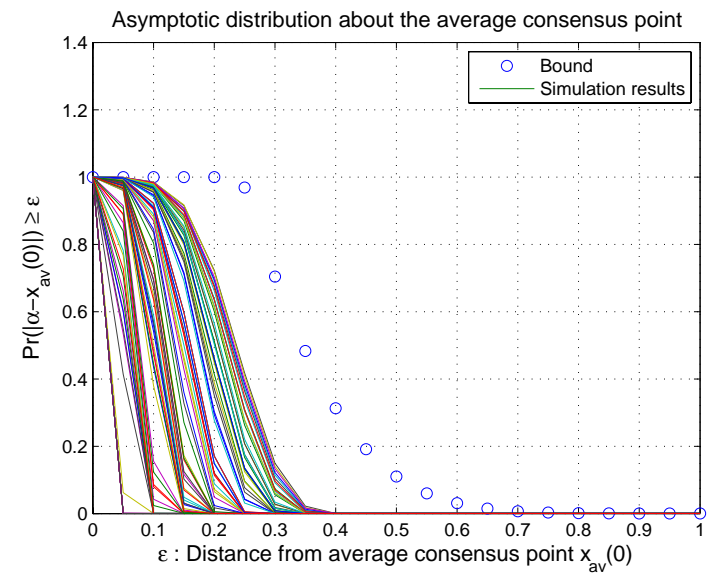

Fig. 2. Simulation results for $N=2$ nodes running the average consensus algorithm over a fading channel. The plot here shows the distance from the consensus value from the average consensus value for a range of initial states $x(0)$. These results are compared with the theoretically obtained bound in Proposition 3.3.

with parameters $p_{12}=0.2$ and $p_{21}=0.8$ for links $2 \rightarrow 1$ and $1 \rightarrow 2$ respectively. The packet losses are assumed to be independent of each other and across time. The initial values are chosen to be $x_{0}(0)=0.4$ and $x_{1}(0)$ as uniformly random from -0.4 to 0.4 . Note that our bound depends only on $\|x(0)\|_{\infty}=0.4$ in this example.

To calculate the Monte Carlo estimate of the probability distribution, we run 100 sample runs each starting at a particular initial state chosen as described above. For each sample run, we execute 10000 simulations with the link losses happening probabilistically. The asymptotic limit for each simulation is approximated by running up to 50 iterations. For each sample run (and hence each distinct initial condition), we thus obtain an empirical probability distribution of the distance of the asymptotic value from the average consensus value. For various sample runs, we thus have a family of probability distributions. This family is compared with the theoretical bound in Figure 2. We see that even for this simple network, the observed asymptotic consensus value has a broad support. We also verify that the entire family of observed probability distributions can be bounded by our results.

\section{CONCLUSIONS}

In this paper, we have studied the probabilistic evolution of a network of agents implementing the average consensus algorithm. While the fact that these system achieve consensus is known, our contribution is new concentration bounds on the state evolution at any time. These results are used to characterize the asymptotic distribution of the consensus point about the average consensus point. Our formulation allows the analysis of consensus algorithms over time-varying fading and interference-limited wireless networks with more realistic assumptions than before.

\section{REFERENCES}

[1] J. N. Tsitsiklis, Problems in Decentralized Decision Making and Computation. PhD thesis, Department of EECS, MIT, Nov. 1984.

[2] V. Blondel, J. Hendrickx, A. Olshevsky, and J. Tsitsklis, "Convergence in multiagent co-ordination, consensus and flocking," Proc. IEEE Conf. Dec. and Control, pp. 2996-3000, 2005.

[3] L. Fang and P. Antsaklis, "On communication requirements for multiagent consensus seeking," Proc. NESC05: Univ. of Notre Dame, Lecture Notes in Control and Information Sciences (LNCIS), Springer, vol. 331, pp. 53-68, 2006.

[4] A. Jadbabaie, J. Lin, and A. S. Morse, "Co-ordination of groups of mobile autonomous agents using nearest neighbor rules," IEEE Trans. Auto. Control, vol. 48(6), pp. 998-1001, June 2003.

[5] L. Moreau, "Consensus seeking in multiagent systems using dynamically changing interconnection topologies," IEEE Trans. Auto. Control, vol. 50(2), 2005.

[6] R. Olfati-Saber and R. M. Murray, "Consensus problems in networks of agents with switching topology and time-delays," IEEE Transactions on Automatic Control, vol. 43, pp. 1520-33, 2004.

[7] D. B. Kingston and R. W. Beard, "Discrete-time average-consensus under switching network topologies," Proc. Am. Control Conf., pp. 3551-3556, 2006.

[8] S. Boyd, A. Ghosh, B. Prabhakar, and D. Shah, "Randomized gossip algorithms," IEEE Trans. Info. Theory, vol. 52(6), pp. 2508-2530, June 2006.

[9] S. Kar and J. M. F. Moura, "Sensor networks with random links: Topology design for distributed consensus," IEEE Trans. Sig. Proc., vol. 56 (7), pp. 3315-3326, July 2008.

[10] A. Tahbaz-Salehi and A. Jadbabaie, "A necessary and sufficient condition for consensus over random networks," IEEE Trans. Auto. Control., vol. 53 (3), pp. 791-795, April 2008.

[11] F. Fagnani and S. Zampieri, "Randomized consensus algorithms over large scale networks," IEEE J. Sel. Areas. Comm., vol. 26 (4), pp. 634-49, May 2008 .

[12] M. Porfiri and D. J. Stilwell, "Consensus seeking over random weighted directed graphs," IEEE Trans. Automatic Control, vol. 52, No. 9, pp. 1767-73, Sept. 2007.

[13] Y. Hatano and M. Mesbahi, "Agreement over random networks," IEEE Trans. Automatic Control, vol. 50, No. 11, pp. 1867-72, Nov. 2005.

[14] R. Carli, F. Fagnani, A. Speranzon, and S. Zampeiri, "Communication constraints in the average consensus problem," Automatica, vol. 44 (3), pp. 671-84, March 2008.

[15] A. Nedich and A. Ozdaglar, "Convergence rate for consensus with delays," LIDS Technical Report 2774, MIT Lab. for Inf. and Dec. Systems.

[16] M. Huang and J. H. Manton, "Stochastic double array analysis and convergence of consensus algorithms with noisy measurements," Proc. Am. Control Conf., pp. 705-710, July 2007.

[17] P. Hovareshti, V. Gupta, and J. S. Baras, "Average consensus over small world networks: A probabilistic framework," IEEE Conf. Dec. and Control, p. Accepted., 2008.

[18] S. Vanka, V. Gupta, and M. Haenggi, "Power-delay analysis of consensus algorithms on wireless networks with interference," Int. J. Sys. Control, and Comm., Spl. Issue on Information Processing and Decision Making in Distributed Control, 2008 (To Appear).

[19] L. Xiao and S. Boyd, "Fast linear iterations for distributed averaging," Systems and Control Letters, vol. 53, pp. 65-78, 2004.

[20] G. B. Folland, Real Analysis: Modern Techniques and Their Applications. Wiley-Interscience, 1999.

[21] D. Williams, Probability with Martingales. Cambridge University Press, 1991.

[22] M. Petkovsek, H. S. Wilf, and D. Zeilberger, $A=B . \quad$ A $\quad \mathrm{K}$ Peters Ltd., 1996. Available online at http://www.math.upenn.edu/ wilf/AeqB.html. 